\title{
Primary culture of chondrocytes after collagenase IA or II treatment of articular cartilage from elderly patients undergoing arthroplasty
}

\author{
Liuliu Xiong ${ }^{1 \# \odot}$, Meng Cui ${ }^{1 \# \oplus}$, Ziye Zhou ${ }^{1 \# \odot}$, Minchen Wu ${ }^{1 \odot}$, Quanming Wang ${ }^{2, * \odot}$, Haiyan Song ${ }^{3 \odot}$, \\ Lei Ding ${ }^{1, *(1)}$
}

Abstract

Background: Joint replacement surgery provides articular cartilage samples for chondrocyte isolation. To our knowledge, the effect of the collagenase type on releasing of chondrocytes from the extracellular matrix of cartilage is not reported.

Objectives: To determine whether cartilage digested with collagenase IA yielded more chondrocytes than that digested with collagenase II and determine whether chondrocytes isolated with collagenase IA could be cultured in vitro.

Methods: Cartilage slices collected from 18 elderly patients who received joint replacement surgery (16 hips, 2 knees) were digested sequentially with $0.4 \%$ pronase $\mathrm{E}$ and $0.02 \%$ collagenase IA, or with $0.15 \%$ collagenase II alone, or sequentially with $0.4 \%$ pronase $\mathrm{E}$ and $0.02 \%$ collagenase II. We compared cell yield from each method. Cell viability by the most effective method was calculated and plotted. The morphology of cultured monolayer chondrocytes was recorded with a light microscope.

Results: Sequential digestion with pronase $\mathrm{E}$ and collagenase IA yielded $2566 \pm 873$ chondrocytes per mg wet cartilage, which was more effective than the other isolation methods $(P=0.018)$. The average chondrocyte viability could reach $84 \% \pm 8 \%(n=11)$. Light microscopic images showed typical chondrocyte morphology in monolayer cultures.

Conclusion: Sequential digestion of human articular cartilage with pronase E and collagenase IA was more effective than collagenase II alone or collagenase II combined with pronase E for releasing chondrocytes from extracellular matrix of cartilage. Chondrocytes isolated with this method could be maintained in monolayer cultures for at least 2 passages with unaltered morphology.

Keywords: arthroplasty, replacement, knee, hip; cartilage, articular; chondrocytes; collagenases; primary cell culture

Arthritis is a degenerative disease affecting articular surfaces of joints. Osteoarthritis and rheumatoid arthritis are 2 major types, both affecting weight-bearing joints or synovial joints, such as knees and hips, which results in edema, pain, stiffness, and even loss of motion. The symptoms are mainly caused by the degradation of hyaline cartilage, the tissue lining the ends of bones

*Correspondence to: Lei Ding, Jiangnan University Wuxi College of Medicine, Wuxi, Jiangsu 214122, China,

email: dinglei@jiangnan.edu.cn or Quanming Wang, Department of Orthopaedic Surgery, Jiangnan University Affiliated Hospital, Wuxi, Jiangsu 214062, China, email: wxsygk@163.com

\#These authors contributed equally to this work.

'Department of Basic Medical Sciences, Jiangnan University Wuxi College of Medicine, Jiangsu 214122, China

${ }^{2}$ Department of Orthopaedic Surgery, Jiangnan University Affiliated Hospital, Jiangsu 214062, China

${ }^{3}$ Department of Endocrinology and Metabolism, The Second Affiliated Hospital of Harbin Medical University, Harbin 150001, China

O Open Access. ( 2021 Xiong et al., published by Sciendo. (c) BY-NC-ND This work is licensed under the Creative Commons Attribution NonCommercial-NoDerivatives 4.0 License. 
inside a synovial joint. Inflammation plays a critical role in cartilage degradation observed in rheumatoid arthritis, which is an autoimmune disorder, and in osteoarthritis, which is associated with aging and mechanical injury of the joint [1,2].

Hyaline cartilage uniquely contains only one cell type, namely chondrocytes. Unlike most cell types in the human body, each individual chondrocyte is housed in a lacuna, which forms a chondron. Chondrons containing chondrocytes and pericellular matrix are interspersed in an extracellular matrix (ECM), which is composed mainly of type II collagen fibers and proteoglycans. Type II collagen fibers are a right-handed triple helix of 3 identical $\alpha 1$ polypeptide chains, each of which forms an extended left-handed helix. Proteoglycans are formed by a core protein and many glycosaminoglycans [3].

Due to the anionic nature of glycosaminoglycans, water molecules are attracted to proteoglycans. Hydroxylysine and glucosyl and galactosyl side chains of type II collagen connect this macromolecule to proteoglycans in the ECM of hyaline cartilage, which enables the tissue to act as a cushion for impact by absorbing and expelling water to protect a synovial joint from injuries during repetitive loading motions [4]. In addition to type II collagen, pericellular matrix contains type VI and IX collagen [5].

Cartilage ECM proteins, mainly proteoglycans and collagen, are synthesized and secreted by chondrocytes. This makes those cells the target for tissue-engineered cartilage repair and for studying the pathogenesis of cartilage degeneration $[6,7]$. Both purposes require the isolation of chondrocytes from cartilage ECM and culturing of isolated cells in vitro. Based on the structural properties of cartilage, proteinases that can cleave collagen and the core protein of proteoglycans are required to loosen the ECM and then liberate chondrocytes from the ECM.

Digestion of the collagen network in cartilage ECM can be achieved by the action of collagenases, which specifically cleave the covalent bond between Gly and a neutral amino acid residue $(\mathrm{X})$ in the sequence of Pro-X-Gly-Pro. This sequence is found in high frequency in polypeptides that form collagen $[8,9]$. Commercially available collagenases are purified from the fermentation of Clostridium histolyticum. According to their effectiveness in dissociating specific tissue types, collagenases are divided into several types. Collagenase II (catalog No. C6885) from Sigma-Aldrich is suitable for isolation of fat cells from adipose tissue and cells from epithelium. This type of collagenase has been widely used to isolate chondrocytes from human cartilage [10-14].

To repair cartilage defects with autologous chondrocytes, Brittberg et al. first harvested cartilage slices from a minor loadbearing area on the upper medial femoral condyle of the injured knee. Next, they incubated minced cartilage with $0.1 \%$ collagenase II for $16 \mathrm{~h}$ to isolate chondrocytes. Other studies have reported using collagenase II with concentrations varying from $0.08 \%$ to $0.3 \%$ and incubation times varying from 16 to $36 \mathrm{~h}$ to isolate chondrocytes from human septal or articular cartilage $[15,16]$.

However, very few studies have examined the effecticacy of collagenase IA for chondrocyte isolation from human cartilage. This type of collagenase is encoded by $\operatorname{col} G$, which is distinct from collagenase II encoded by colH. Compared to collagenase II, collagenase IA has higher collagenase activity and is more efficient in digesting human adult and fetal brain, lung, and tumor tissues $[17,18]$. Furthermore, collagenase IA was routinely used in our previous studies for isolation of chondrocytes from bovine articular cartilage and once used for isolation of human articular chondrocytes from amputated ankle joint of a young patient [19]. Based on our previous experience, we hypothesized that collagenase IA was more effective than collagenase II to isolate chondrocytes from human articular cartilage obtained from synovial joints of elderly patients who underwent total joint replacement surgery. Our aims were to determine whether (1) cartilage digested with collagenase IA yielded more chondrocytes than that digested with collagenase II; and (2) whether chondrocytes isolated with collagenase IA could be cultured in vitro.

\section{Methods}

\section{Patient characteristics and cartilage yield}

Eighteen patients ( 55 to 87 years, 12 women, 6 men) who were diagnosed with femoral neck fracture (11 patients), femoral head necrosis (4 patients), and hip (1 patient) or knee ( 2 patients) osteoarthritis underwent total hip or knee replacement surgery at the Department of Orthopaedic Surgery of Jiangnan University Affiliated Hospital. The removed femoral head, femoral condyles, and tibial plateau, regarded as surgical waste, were collected with approval of the institutional review board of Jiangnan University Affiliated Hospital (approval No. LS2019001) and with documented informed consent from each donor patient. The study conducted in accordance with the principles outlined in the contemporary revision of the Declaration of Helsinki of 1964 (World Medical Association) incorporating the most recent (2013) and earlier amendments. Patients were divided into 3 groups based on age, sex, and diagnosis. The number of patients in each subgroup and the yield of cartilage are summarized in Table 1.

\section{Harvest of cartilage from osteochondral specimens}

Upon completion of each total hip or knee replacement surgery, osteochondral specimens (femoral head or condyles or tibial plateau) were saved in sterile normal saline and transferred 
from the operation room to the laboratory in a cold bag. Inside a BSL-2 biosafety cabinet, full thicknesses of cartilage slices were shaved aseptically from the osteochondral specimen using a size 20-24 surgical blade. Cartilage slices were weighed and then cut into 5-10 $\mathrm{mm}$ pieces before being transferred into a sterile spinner flask (Wheaton) for enzymatic treatments.

\section{Enzymatic dissociation of chondrocytes from cartilage}

To compare the effectiveness of enzymatic treatments, cartilage slices were divided into 3 groups: (1) Group I, treated with $0.4 \%$ (weight to volume ratio [w/v]) pronase E (Sigma-Aldrich, catalog No. P5147) for $90 \mathrm{~min}$ and then with $0.02 \%(\mathrm{w} / \mathrm{v}) \mathrm{col}-$ lagenase IA (Sigma-Aldrich, catalog No. C9891) for 16 h; (2) Group II, treated with $0.15 \%$ (w/v) collagenase II (catalog No. A004174, Sangon Biotech, Shanghai, China) only for $16 \mathrm{~h}$; and (3) Group III, treated with $0.4 \%(\mathrm{w} / \mathrm{v})$ pronase E for $90 \mathrm{~min}$ and then with $0.02 \%(\mathrm{w} / \mathrm{v})$ collagenase II for $16 \mathrm{~h}$. For each group, characteristics of donor patients are listed in Table 2. All enzymatic treatments were conducted in a sterile spinner flask sitting on a magnetic stirrer specifically designed for cell isolation (DLAB Scientific) placed inside a tissue culture incubator $\left(37^{\circ} \mathrm{C}, 5 \% \mathrm{CO}_{2}, 100 \%\right.$ humidity). The stirring speed was set at $70 \mathrm{rpm}$ over the course of enzymatic treatments.

\section{Chondrocyte yield and viability}

After cartilage slices were digested with collagenase IA or II, the cell suspension was first filtered with a cell strainer (pore size: $70 \mu \mathrm{m})$ to remove undigested tissue debris and then centrifuged at $1000 \mathrm{rpm}$ for $8 \mathrm{~min}$ at room temperature. Cell pellets were then resuspended with Dulbecco's modified Eagle's medium supplemented with Nutrient Mixture F-12 (DMEM/F12) supplemented with $10 \%$ fetal bovine serum (FBS) and $1 \%$ penicillin-streptomycin. Cells were stained with $0.4 \%$ trypan blue. Total cell number and cell viability were determined using a hemocytometer. Chondrocyte yield was computed by dividing total cell number with the wet weight of cartilage harvested.

\section{Chondrocyte monolayer cultures}

Isolated chondrocytes were seeded at $2.5 \times 10^{5}$ cells $/ \mathrm{cm}^{2}$ in a treated 12 well tissue-culture plate and cultured in a $37^{\circ} \mathrm{C}$ cell culture incubator under an atmosphere of $5 \% \mathrm{CO}_{2}$ at $100 \%$ humidity. Culture media were changed every 2 days. Chondrocytes were examined daily using an Olympus CKX53 light microscope equipped with $4 \times, 10 \times$, and $20 \times$ objectives. Light microscopic images of chondrocytes in monolayers were recorded with a digital camera (Olympus LC30) installed on the microscope. We selected without preference 3 areas of chondrocyte monolayers in each well of a plate for imaging.

\section{Statistical analyses}

Chondrocyte yield in each group was averaged and standard deviation (SD) was calculated. We used Microsoft Excel for Mac (version 16.16.27 (201012)) for statistical analyses. The mean of chondrocyte yield between the 3 groups was compared with a $t$ test (2-sample assuming unequal variances) commonly known as a Welch a $t$ test or a Welch unequal variances

Table 1. Donor patient characteristics and wet weights of cartilage harvested

\begin{tabular}{|c|c|c|c|c|}
\hline \multicolumn{2}{|c|}{ Patient characteristic } & \multirow{2}{*}{$\frac{\text { No. of patients }}{1}$} & \multirow{2}{*}{$\frac{\% \text { of total patients }}{6}$} & \multirow{2}{*}{$\begin{array}{c}\begin{array}{c}\text { Mean yield of wet cartilage } \pm \text { SD } \\
\text { (mg) }\end{array} \\
3430\end{array}$} \\
\hline Age (y) & $50-59$ & & & \\
\hline & $60-69$ & 6 & 33 & $3760 \pm 1489$ \\
\hline & 70-79 & 8 & 44 & $4211 \pm 1716$ \\
\hline & $80-89$ & 3 & 17 & $2740 \pm 1221$ \\
\hline \multirow[t]{2}{*}{ Sex } & Male & 6 & 33 & $3708 \pm 1035$ \\
\hline & Female & 12 & 67 & $3804 \pm 1781$ \\
\hline \multirow[t]{4}{*}{ Diagnosis } & Femoral neck fracture & 11 & 61 & $3245 \pm 1137$ \\
\hline & Femoral head necrosis & 4 & 22 & $3253 \pm 765$ \\
\hline & Hip osteoarthritis & 1 & 6 & 6450 \\
\hline & Knee osteoarthritis & 2 & 11 & 6370 \\
\hline
\end{tabular}

SD, standard deviation. 
Table 2. Donor patient characteristics and wet weight of harvested cartilage for each treatment group

\begin{tabular}{|c|c|c|c|c|c|}
\hline $\begin{array}{l}\text { Group No. } \\
\text { (cartilage treatment; sample size) }\end{array}$ & Patient No. & Age (y) & Sex & Diagnosis & $\begin{array}{l}\text { Wet weight of cartilage } \\
(\mathrm{mg})(\text { mean } \pm \text { SD) }\end{array}$ \\
\hline \multirow[t]{11}{*}{ I (Pronase E + Collagenase IA; $\mathrm{n}=11)$} & 1 & 76 & $\mathrm{~F}$ & Left femoral neck fracture & $4396 \pm 1546$ \\
\hline & 2 & 62 & M & Right femoral head necrosis & \\
\hline & 3 & 62 & $\mathrm{~F}$ & Right femoral head necrosis & \\
\hline & 4 & 81 & $\mathrm{~F}$ & Right femoral neck fracture & \\
\hline & 5 & 77 & M & Left femoral neck fracture & \\
\hline & 6 & 62 & $\mathrm{~F}$ & Left femoral neck fracture & \\
\hline & 7 & 61 & $\mathrm{~F}$ & Right knee osteoarthritis & \\
\hline & 8 & 74 & M & Left femoral neck fracture & \\
\hline & 9 & 75 & $\mathrm{~F}$ & Left knee osteoarthritis & \\
\hline & 10 & 70 & $\mathrm{~F}$ & Right hip osteoarthritis & \\
\hline & 11 & 79 & $\mathrm{~F}$ & Left femoral neck fracture & \\
\hline \multirow[t]{5}{*}{ II (Collagenase II; $\mathrm{n}=5$ ) } & 12 & 83 & $\mathrm{~F}$ & Left femoral neck fracture & $2854 \pm 876.5$ \\
\hline & 13 & 67 & $\mathrm{~F}$ & Right femoral head necrosis & \\
\hline & 14 & 55 & M & Right femoral head necrosis & \\
\hline & 15 & 72 & $\mathrm{~F}$ & Right femoral neck fracture & \\
\hline & 16 & 87 & M & Left femoral neck fracture & \\
\hline \multirow[t]{2}{*}{ III (Pronase E + Collagenase II; n= 2) } & 17 & 64 & M & Left femoral neck fracture & 2640 \\
\hline & 18 & 72 & $\mathrm{~F}$ & Left femoral neck fracture & \\
\hline
\end{tabular}

SD, standard deviation; $M$, male; F, female.

$t$ test. Chondrocyte viability was averaged from 11 batches of experiments in which pronase E and collagenase IA were employed to isolate chondrocytes from cartilage specimens. SD of chondrocyte viability was also calculated. Differences with $P<0.05$ were considered significant.

\section{Results}

\section{Patient characteristics and cartilage yield}

Among 18 patients who provided osteochondral specimens for the study, those in the group aged 70-79 years yielded the most cartilage, with an average wet weight of $4211 \pm 1716 \mathrm{mg}$. Cartilage yield from male patients was slightly lower than that from female patients. Cartilage yield from the femoral neck fracture group was almost equal to that from the femoral head necrosis group (Table 1).

\section{Enzymatic digestion of cartilage}

After $16 \mathrm{~h}$ incubation with collagenase II in Group II or III, cartilage slices were still visible to the naked eye. The number and size of cartilage slices did not reduce remarkably compared with that at the beginning of the enzymatic digestion process. However, compared with those in Groups II or III, cartilage slices in Group I, which were first digested with $0.4 \%$ pronase $\mathrm{E}$ for $90 \mathrm{~min}$ and then with $0.02 \%$ collagenase IA for $16 \mathrm{~h}$, were barely visible to the naked eye at the end of enzymatic digestion process.

\section{Chondrocyte yields}

After collagenase digestion, samples in Group I (pronase E + collagenase IA) produced the most chondrocytes $(P=0.018)$ compared with Group II, in which cartilage slices were digested with collagenase II alone. Cartilage slices in Group III, which were subjected to pronase E digestion and then to collagenase II digestion, yielded the least chondrocytes (Figure 1). Each group was categorized further into 2 sets of subgroups based on the donor patient age, sex, and diagnosis, and the corresponding chondrocyte yield is listed (Table 3). Chondrocyte yield for Groups II or III was substantially lower than that in Group I regardless of subgroup.

\section{Chondrocytes isolated from Group I had high viability}

Freshly isolated chondrocytes in Group I were stained with trypan blue to identify dead cells. Cell viability in each 


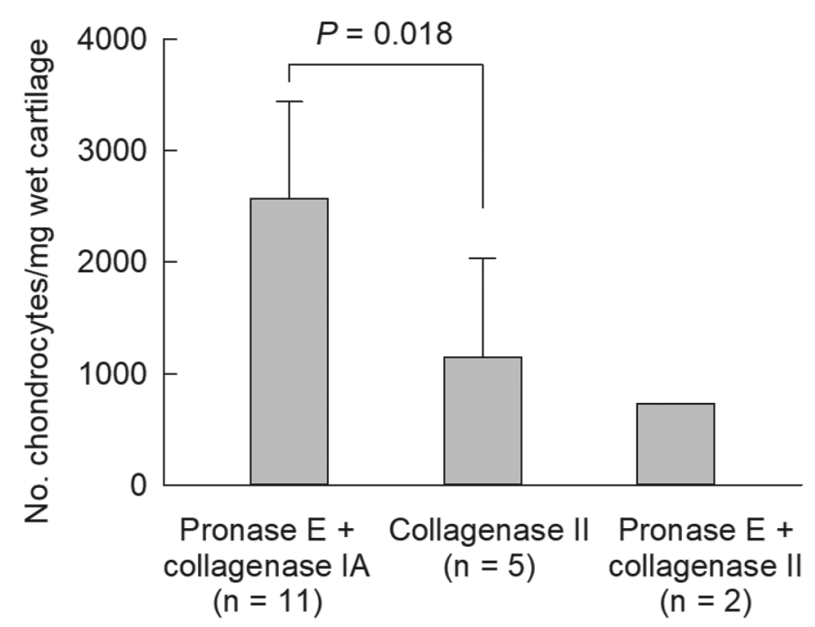

Figure 1. Comparison of cell yield between 3 chondrocyte isolation methods. Chondrocytes were isolated from elderly human articular cartilage using 3 methods. We divided cartilage slices in 3 groups: Group I, digested sequentially with pronase E and collagenase IA; Group II, digested with collagenase II only; or Group III, digested sequentially with pronase $\mathrm{E}$ and collagenase II. After digestion, total cell number was counted and mean cell yield (No. of chondrocytes per mg wet weight of cartilage) is plotted. We compared the difference in cell yield between Groups I and II with a 2-tailed $t$ test assuming unequal variances. Error bars represent SD. SD, standard deviation.

batch of chondrocyte isolation experiments was calculated and plotted. Among 11 cartilage specimens that were subjected to pronase $\mathrm{E}$ and collagenase IA digestion, the highest viability of isolated chondrocytes was $95 \%$ from specimen No. 1 while the lowest viability was $66 \%$ from specimen No. 7. The majority of specimens showed chondrocyte viability between $75 \%$ and $92 \%$. The mean and SD of chondrocyte viability from all batches were $84 \% \pm 8 \%$ (Figure 2).

Chondrocytes isolated from Group I were seeded at high density in a 12-well tissue culture plate and cultured in DMEM/F12 supplemented with $10 \%$ FBS. Two days after seeding, cells reached about $80 \%$ confluence and the majority displayed a spherical or elliptical shape, which is a typical phenotype of articular chondrocytes. Less than $10 \%$ of the cells were fully attached to the culture plate and showed a stretched irregular shape (Figures 3A and B). When passage 0 cells were cultured for 10 days in monolayers, most cells still maintained an elliptical shape and a small fraction showed a spreading fibroblastic morphology (Figure 3C). One day after subculturing, the majority of passage 1 cells remained spherical or elliptical. Less than $10 \%$ of the cells displayed a fibroblastic morphology (Figure 3D).

\section{Discussion}

Results presented in this report support our hypothesis that collagenase IA is more effective than collagenase II to isolate chondrocytes from articular cartilage of synovial joints from elderly patients who had undergone total joint replacement surgery.

Those chondrocytes isolated using collagenase IA digestion were highly viable and could be cultured in vitro for 2 passages without changes in chondrocyte morphology. Using sequential digestion with pronase $\mathrm{E}$ and collagenase IA, we could isolate approximately 2.5 million chondrocytes from $1 \mathrm{~g}$ of wet cartilage. The average viability of those isolated chondrocytes was around $84 \%$, which was higher than that reported by Jakob et al. after a study using various protocols based on combinations of collagenase II, trypsin, hyaluronidase, and tosyl-lysyl chloromethane [13]. To our knowledge, this is the first report of a study to examine directly the difference in human chondrocyte isolation efficiency between widely used collagenase II and rarely used collagenase IA.

As a pathogenic bacterium, Clostridium histolyticum can cause myonecrosis by producing collagenase, a zinc-containing metalloproteinase, to dissolve various types of collagen. Compared with collagenases produced by vertebrates, collagenase from $C$. histolyticum has a broader substrate specificity and more potent collagenolytic activity [17]. Collagenase IA or collagenase type I, is composed of several collagenase proteins, some of which are $\mathrm{C}$-truncated forms, all of which are encoded by $\operatorname{colG}$. The product of this gene is mature collagenase IA with a molecular weight around $114 \mathrm{kDa}$. By contrast, collagenase II is encoded by $\mathrm{colH}$ and is composed of a mature protein with a molecular weight of $112 \mathrm{kDa}$ and one $\mathrm{C}$-truncated form [17, 20].

Our data indicate that collagenase IA is more suitable than collagenase II for isolating chondrocytes from human cartilage. Bond and Wart found that collagenase IA contained predominantly $\beta$-collagenase, while collagenase II only contained a trace. $\beta$-Collagenase is highly active against collagen [18]. To maximize the effect of collagenase IA, cartilage needs to be pretreated with pronase E, mainly to digest proteoglycans, which can expose sites on collagen for collagenase IA to bind. Pronase E is a mixture of 10 proteases that are produced by Streptomyces griseus K-1. Narahashi and Yanagita showed that pronase E was much more effective in digesting casein than trypsin because the former contained serine proteases and peptidases [21, 22].

Comparing chondrocyte yield and examining cell viability, we identified the sequential digestion of cartilage with pronase E and collagenase IA as the most effective way to isolate chondrocytes from human cartilage. The sequential digestion method involving pronase and collagenase has also been used by Diaz-Romero et al. to isolate chondrocytes 
Table 3. Donor patient characteristics and chondrocyte yield for each treatment group

\begin{tabular}{|c|c|c|c|c|}
\hline \multirow{2}{*}{$\begin{array}{l}\text { Treatment } \\
\text { Group I }(n=11) \text { : pronase }\end{array}$} & \multicolumn{2}{|c|}{ Characteristic } & \multirow{2}{*}{$\frac{\text { No. of patients }}{4}$} & \multirow{2}{*}{$\begin{array}{c}\begin{array}{c}\text { Chondrocyte yield } \\
\text { (mean cells } \pm \text { SD) }\end{array} \\
2940 \pm 629\end{array}$} \\
\hline & Age (y) & $60-69$ & & \\
\hline $\mathrm{E}+$ collagenase IA & & $70-79$ & 6 & $2390 \pm 1050$ \\
\hline & & $80-89$ & 1 & 2130 \\
\hline & Sex & Male & 3 & $2514 \pm 601$ \\
\hline & & Female & 8 & $2586 \pm 992$ \\
\hline & Diagnosis & Femoral neck fracture & 6 & $2587 \pm 1056$ \\
\hline & & Femoral head necrosis & 2 & 3002 \\
\hline & & Hip osteoarthritis & 1 & 3030 \\
\hline & & Knee osteoarthritis & 2 & 1837 \\
\hline \multirow[t]{8}{*}{ Group II $(n=5)$ : collagenase II } & Age (y) & $50-59$ & 1 & 2729 \\
\hline & & $60-69$ & 1 & 747 \\
\hline & & $70-79$ & 1 & 669 \\
\hline & & $80-89$ & 2 & 789 \\
\hline & Sex & Male & 2 & 1702 \\
\hline & & Female & 3 & $773 \pm 119$ \\
\hline & Diagnosis & Femoral neck fracture & 3 & $749 \pm 133$ \\
\hline & & Femoral head necrosis & 2 & 1738 \\
\hline \multirow{5}{*}{$\begin{array}{l}\text { Group III }(n=2) \text { : pronase } \\
E+\text { collagenase II }\end{array}$} & Age (y) & $60-69$ & 1 & 1291 \\
\hline & & $70-79$ & 1 & 170 \\
\hline & Sex & Male & 1 & 1291 \\
\hline & & Female & 1 & 170 \\
\hline & Diagnosis & Femoral neck fracture & 2 & 730 \\
\hline
\end{tabular}

SD, standard deviation.

from human cartilage of femoral condyles. Our findings were consistent with those of these authors, who reported that the method produced a better chondrocyte yield than collagenase alone [23]. However, Diaz-Romero et al. did not specify the type of pronases they used in their study. Collagenase $\mathrm{P}$ was used for the second step of enzymatic dissociation. This type of collagenase acts more like collagenase II than I. Because the authors did not provide the actual chondrocyte yields from their study and use cartilage specimens from donors with a wide age range (32-89 years old), direct comparison of chondrocyte yields between their method and ours are difficult.

In published studies on human chondrocyte isolation, protocol specificities, including the concentrations of proteinases, incubation times, and the order in which different proteinases were applied, vary widely. In a study using autologous chondrocytes for articular cartilage restoration in patients with age ranging from 14 to 48 years, cartilage obtained from a minor load-bearing joint was digested with $0.1 \%$ collagenase (w/v) for $16 \mathrm{~h}$. Around 900 chondrocytes were isolated from $1 \mathrm{mg}$ of cartilage [15]. Although the authors did not indicate the type of collagenase used in their study, their chondrocyte yield was less than half of what we observed in Group I in our study despite that they used cartilage specimens from much younger patients and 5 times greater concentration of collagenase.

Yonenaga et al. collected cartilage specimens from 3 elderly patients diagnosed with osteoarthritis and tested a series of collagenase concentration and incubation times to identify optimal conditions for articular chondrocyte isolation. They reported that $0.6 \%$ collagenase with $24 \mathrm{~h}$ 
incubation were optimal conditions, yielding around 1000 viable chondrocytes per mg of wet cartilage [24]. However, the authors did not specify which type of collagenase they

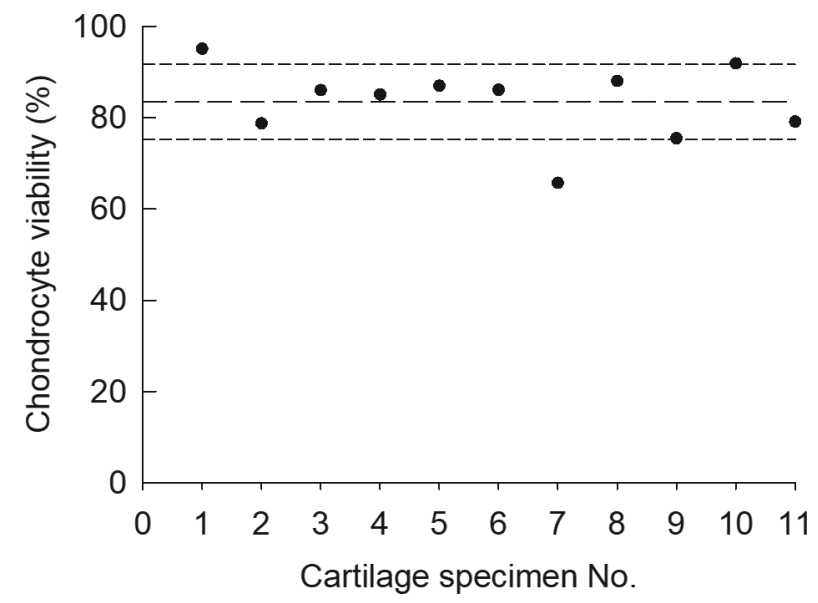

Figure 2. Viability of chondrocytes isolated from 11 cartilage specimens using pronase $E$ and collagenase IA. Cartilage specimens obtained from 11 patients were first incubated with $0.4 \%$ pronase $E$ and then with $0.02 \%$ collagenase IA. The viability of chondrocytes isolated from each patient is plotted. The long dashed line indicates the mean level of chondrocyte viability (84\%) computed from 11 specimens. The short dashed lines indicate one standard deviation from the mean (8\%).
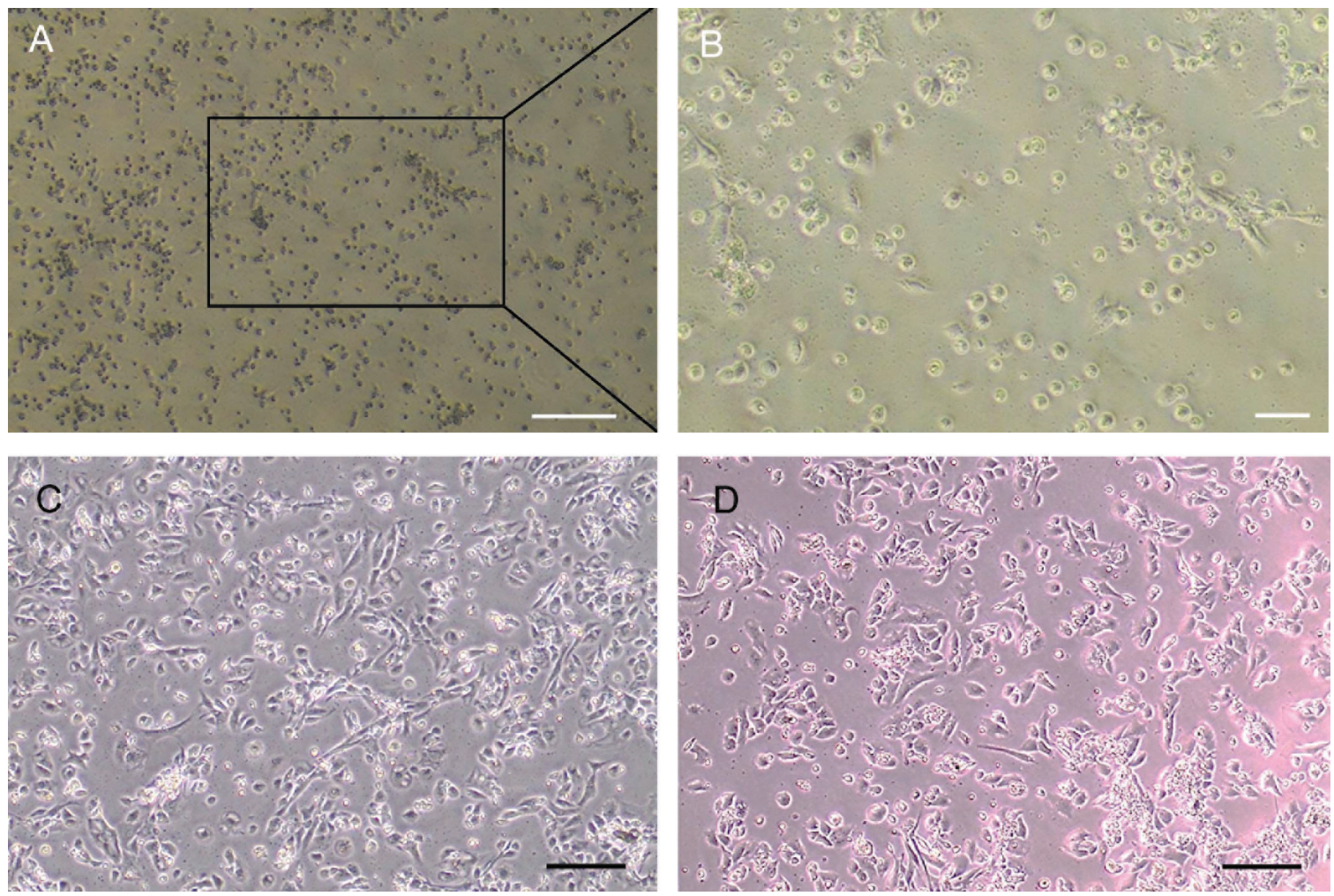

Figure 3. Representative light microscopic images of monolayer human chondrocytes. (A) Passage 0 human chondrocytes isolated with pronase E and collagenase IA seeded at $2.5 \times 10^{5}$ cells per $\mathrm{cm}^{2}$ and visualized at day 2 post-seeding ( $4 \times$ objective lens, scale bar $\left.200 \mu \mathrm{m}\right)$ and (B) detail of the insert in the black square as imaged with a $10 \times$ objective (scale bar $50 \mu \mathrm{m}$ ). (C) Passage 0 chondrocytes at day 10 post-seeding imaged with a $4 \times$ objective (scale bar $200 \mu \mathrm{m}$ ). (D) Passage 1 chondrocytes at day 1 post-seeding imaged with a $10 \times$ objective (scale bar $200 \mu \mathrm{m}$ ). used in their study or the exact anatomical locations from which they harvested cartilage specimens. Possibly because of the higher collagenase concentration and longer incubation time used, their viable chondrocyte yield was lower than the yield that we observed in Group I. Moreover, our results were derived from 11 cartilage specimens obtained from elderly patients of both sexes, while their study used pooled cartilage specimens obtained from only 3 elderly female patients.

We acknowledge that cartilage in 3 enzymatic treatment groups - is not from the same patient-and this may complicate the interpretation of chondrocyte yield data due to individual variation. The reason why we did not use the same batch of cartilage to compare chondrocyte yield among 3 enzymatic methods was that we could only harvest around $3.8 \mathrm{~g}$ of wet cartilage from 1 human femoral head and when we divided this amount of cartilage into 3 enzymatic treatment groups, the total number of chondrocytes isolated in each group was too low to be counted accurately. This may be the result of the uniqueness of cartilage tissue, including its relatively high ECM to cell volume ratio, and that only $10 \%$ of tissue volume is occupied by chondrocytes [25].

Cartilage samples from more than 1 donor patient could not be pooled because we could only receive 1 femoral head 
or tibial plateau specimen on a certain day, which was determined by patient enrolment and surgery schedule. We could not refrigerate previously received specimens because refrigeration would cause time-dependent chondrocyte death in cartilage [26] and we could not predict when the next specimens would be collected. Moreover, according to the report by Stockwell, during aging (30-89 years), cell density in human articular cartilage remained constant (at about 10 cells per 0.22 $\mathrm{mm}^{2}$ ) [27]. This implies that the number of chondrocytes in $1.0 \mathrm{mg}$ wet weight of cartilage (chondrocyte yield) should be independent of age and sex of patients who provided femoral head or tibial plateau cartilage for our study. We observed that chondrocyte yield in Group I was significantly higher than that in Group II or III. This indicates that collagenase IA is more effective than collagenase II in releasing chondrocytes from articular cartilage.

Here, we developed an effective protocol for chondrocyte isolation from the articular cartilage of elderly patients. Chondrocyte yield and viability were superior to that described for other studies using human cartilage to isolate chondrocytes. Because total hip or knee replacement surgery provides the vast majority of human cartilage specimens in clinics, our chondrocyte isolation protocol may facilitate studies of the pathogenesis of osteoarthritis and cartilage tissue engineering.

Author contributions: LD contributed the conception and design of the study. LX, MC, and ZZ performed the experiments and analyzed the data. MW, QW, and HS contributed to the experimental design and data analysis. LD supervised the work, drafted the manuscript, and obtained the funding for the project. All authors revised the manuscript for critical intellectual content, approved the final version submitted for publication, and take responsibility for the statements made in the published article.

Acknowledgments: The present study was funded by the National Innovation and Entrepreneurship Program for College Students of China (201810295021) and by the Jiangsu Provincial Natural Science Foundation of China grant awarded to Lei Ding (BK20171143). The authors thank Mr. Dingqi Xie for making the scale bars in light microscopic images more readable.

Conflict of interest statement: The authors have each completed and submitted an International Committee of Medical Journal Editors Disclosure Form for Potential Conflicts of Interest. None of the authors has any potential or actual conflict of interest to disclose that is related to the published article.
Data sharing statement. Data generated and analyzed to support the findings presented in the article are included in this published article as statistical summaries or actual data. Further details are available from the corresponding author on reasonable request after deidentification from any patient whose data are included in this report.

\section{References}

[1] Aspden RM, Saunders FR. Osteoarthritis as an organ disease: from the cradle to the grave. Eur Cell Mater. 2019; 37:74-87.

[2] Croia C, Bursi R, Sutera D, Petrelli F, Alunno A, Puxeddu I. One year in review 2019: pathogenesis of rheumatoid arthritis. Clin Exp Rheumatol. 2019; 37:347-57.

[3] Wilusz RE, Sanchez-Adams J, Guilak F. The structure and function of the pericellular matrix of articular cartilage. Matrix Biol. 2014; 39:25-32.

[4] Mayne R. Cartilage collagens. What is their function, and are they involved in articular disease? Arthritis Rheum. 1989; 32:241-46.

[5] Hagiwara H, Schröter-Kermani C, Merker HJ. Localization of collagen type VI in articular cartilage of young and adult mice. Cell Tissue Res. 1993; 272:155-60.

[6] Evans CH. Catering to chondrocytes. Sci Transl Med. 2018; 10:eaav7043. doi: 10.1126/scitranslmed.aav7043

[7] Wieland HA, Michaelis M, Kirschbaum BJ, Rudolphi KA. Osteoarthritis - an untreatable disease? Nat Rev Drug Discov. 2005; 4:331-44.

[8] Gelse K, Pöschl E, Aigner T. Collagens-structure, function, and biosynthesis. Adv Drug Deliv Rev. 2003; 55:1531-46.

[9] Miller EJ, Harris EJ, Chung E, Finch JJ, McCroskery PA, Butler WT. Cleavage of Type II and III collagens with mammalian collagenase: site of cleavage and primary structure at the $\mathrm{NH}_{2}$-terminal portion of the smaller fragment released from both collagens. Biochemistry. 1976; 15:787-92.

[10] Rotter N, Bonassar LJ, Tobias G, Lebl M, Roy AK, Vacanti CA. Age dependence of cellular properties of human septal cartilage: implications for tissue engineering. Arch Otolaryngol Head Neck Surg. 2001; 127:1248-52.

[11] Tallheden T, van der Lee J, Brantsing C, Månsson JE, SjögrenJansson E, Lindahl A. Human serum for culture of articular chondrocytes. Cell Transplant. 2005; 14:469-79.

[12] Marsano A, Millward-Sadler SJ, Salter DM, Adesida A, Hardingham T, Tognana E, et al. Differential cartilaginous tissue formation by human synovial membrane, fat pad, meniscus cells and articular chondrocytes. Osteoarthritis Cart. 2007; 15:48-58.

[13] Jakob M, Démarteau O, Schäfer D, Stumm M, Heberer M, Martin I. Enzymatic digestion of adult human articular cartilage yields a small fraction of the total available cells. Connect Tissue Res. 2003; 44:173-80.

[14] Dunham BP, Koch RJ. Basic fibroblast growth factor and insulinlike growth factor I support the growth of human septal chondrocytes in a serum-free environment. Arch Otolaryngol Head Neck Surg. 1998; 124:1325-30.

[15] Brittberg M, Lindahl A, Nilsson A, Ohlsson C, Isaksson O, Peterson L. Treatment of deep cartilage defects in the knee with autologous chondrocyte transplantation. N Engl J Med. 1994; 331:889-95. 
[16] Oseni AO, Butler PE, Seifalian AM. Optimization of chondrocyte isolation and characterization for large-scale cartilage tissue engineering. J Surg Res. 2013; 181:41-8.

[17] Matsushita O, Jung C-M, Katayama S, Minami J, Takahashi Y, Okabe A. Gene duplication and multiplicity of collagenases in Clostridium histolyticum. J Bacteriol. 1999; 181:923-33.

[18] Bond MD, Van Wart HE. Purification and separation of individual collagenases of Clostridium histolyticum using red dye ligand chromatography. Biochemistry-US. 1984; 23:3077-85.

[19] Ding L, Buckwalter JA, Martin JA. DAMPs synergize with cytokines or fibronectin fragment on inducing chondrolysis but lose effect when acting alone. Mediators Inflamm. 2017:2642549. doi: 10.1155/2017/2642549

[20] Yoshihara K, Matsushita O, Minami J, Okabe A. Cloning and nucleotide sequence analysis of the $\mathrm{colH}$ gene from Clostridium histolyticum encoding a collagenase and a gelatinase. J Bacteriol. 1994; 176:6489-96.

[21] Sweeney PJ, Walker JM. Pronase (EC 3.4.24.4). Methods Mol Biol. 1993; 16:271-6.
[22] Narahashi Y, Yanagita M. Studies on proteolytic enzymes (pronase) of Streptomyces griseus K-1. I. Nature and properties of the proteolytic enzyme system. J Biochem. 1967; 62:633-41.

[23] Diaz-Romero J, Gaillard JP, Grogan SP, Nesic D, Trub T, MainilVarlet P. Immunophenotypic analysis of human articular chondrocytes: changes in surface markers associated with cell expansion in monolayer culture. J Cell Physiol. 2005; 202:731-42.

[24] Yonenaga K, Nishizawa S, Nakagawa T, Fujihara Y, Asawa Y, Hikita A, et al. Optimal conditions of collagenase treatment for isolation of articular chondrocytes from aged human tissues. Regen Ther. 2017; 6:9-14.

[25] Archer CW, Francis-West P. The chondrocyte. Int J Biochem Cell Biol. 2003; 35:401-4.

[26] Ding L, Zampogna B, Vasta S, Jang KW, De Caro F, Martin JA, Amendola A. Why do osteochondral allografts survive? Comparative analysis of cartilage biochemical properties unveils a molecular basis for durability. Am J Sports Med. 2015; 43:2459-68.

[27] Stockwell RA. The cell density of human articular and costal cartilage. J Anat. 1967; 101:753-63. 